\title{
CONFORMAL UNITARY SPACES
}

BY

\section{N. COBURN}

I. Introduction. In this paper, we shall study the relations between unitary spaces of $n$ dimensions $K_{n}$ and ' $K_{n}$ whose fundamental tensors are related by the conformal transformation.

In $\$ I I$, we develop the notation and some of the fundamental concepts of hermitian geometry. Section III deals with the relation between the paths of conformal unitary spaces. It is shown that (1) two unitary spaces, both without torsion (symmetric connection), cannot be conformal; (2) if two unitary spaces are conformal, then their paths correspond; (3) conversely, if two unitary spaces, one of which is without torsion, are in a restricted correspondence of paths and if an unitary orthogonal ennuple of $K_{n}$ corresponds conformally to an ennuple of ' $K_{n}$ (see Definition 2), and if a certain curvature affinor ${ }^{\prime} C_{\alpha \mu *_{\nu}}^{\cdots \beta}$ vanishes, then the two spaces are conformal.

The paper concludes with a study of some particular conformal unitary spaces. We define the terms: (1) unitary $k$-space; (2) $k$-spaces which are $k$-conformal. We then prove the theorems: (1) the affinor ${ }^{\prime} C_{\alpha \mu *}^{\cdots \beta}$ is a conformal invariant of all $k$-spaces which are $k$-conformal; (2) if two unitary spaces are conformal and the affinor ' $C_{\alpha \mu \mu_{\nu}}^{\cdots \beta}$ is a conformal invariant, then the spaces are $k$-spaces. A particular type of $k$-space with symmetric connection has been studied by Mitrochin, Fuchs, Bergman and others.

II. Notation $\left({ }^{1}\right)$. Consider the "points" determined by assigning arbitrary values to the complex quantities $\xi^{\lambda}\left(x^{\lambda}, y^{\lambda}\right.$ real)

$$
\xi^{\lambda}=x^{\lambda}+i y^{\lambda}, \quad i=(-1)^{1 / 2}, \lambda=1, \cdots, n .
$$

These points build a complex space of $n$ dimensions $X_{n}$ (real topological $\left.\left.X_{2 n}\right){ }^{2}\right)$. Associated with (2.1) are the conjugate quantities

$$
\left(\xi^{\lambda}\right)^{*}=\xi^{\lambda *}=x^{\lambda}-i y^{\lambda}, \quad \lambda^{*}=1, \cdots, n .
$$

Let us denote partial derivatives by

$$
\partial_{\mu}=\partial / \partial \xi^{\mu}, \quad \partial_{\mu^{*}}=\partial / \partial \xi^{\mu^{*}} ;
$$

then from $(2.1)$ by the composite function theorem $\left({ }^{3}\right)$, we find for functions

Presented to the Society, February 24, 1940; received by the editors June 2, 1940.

(1) Since most of the references are to Einführung in die neueren Methoden der Differentialgeometrie by J. A. Schouten and D. J. Struik; vols. 1, 2; P. Noordhoff; Groningen, Batavia; 1935, 1938; we shall merely indicate volume, page and equation number.

(2) II, pp. 225-251.

(3) The equations (2.4) are obtained as follows: 
of $\xi^{\lambda}$ and $\xi^{\lambda^{*}}$ respectively,

$$
\begin{aligned}
\partial_{\mu} \phi\left(\xi^{\lambda}\right) & =\frac{1}{2}\left(\partial \phi / \partial x^{\mu}-i \partial \phi / \partial y^{\mu}\right), \\
\partial_{\mu^{*}} \psi\left(\xi^{\lambda *}\right) & =\frac{1}{2}\left(\partial \psi / \partial x^{\mu}+i \partial \psi / \partial y^{\mu}\right) .
\end{aligned}
$$

Consider the allowable coordinate transformations with nonvanishing Jacobian

$$
\xi^{\lambda}=\xi^{\lambda}\left(\xi^{\lambda^{\prime}}\right), \quad \xi^{\lambda^{\prime}}=x^{\lambda^{\prime}}+i y^{\lambda^{\prime}} .
$$

The corresponding conjugate equations are

$$
\xi^{\lambda^{*}}=\xi^{\lambda^{*}}\left(\xi^{\lambda *^{\prime}}\right), \quad \xi^{\lambda^{\prime}}=x^{\lambda^{\prime}}-i y^{\lambda^{\prime}} .
$$

We now introduce the unit affinor whose intermediary $\left({ }^{4}\right)$ components are

$$
A_{\mu^{\prime}}^{\lambda}=\partial \xi^{\lambda} / \partial \xi^{\mu^{\prime}}, \quad A_{\mu^{* \prime}}^{\lambda^{*}}=\partial \xi^{\lambda^{*}} / \partial \xi^{\mu^{* \prime}} .
$$

Evidently, if a star $\left(^{*}\right)$ after a symbol denotes the conjugate (i.e., replacing $\xi^{\lambda}$ by $\xi^{\eta^{*}}$ and $i$ by $-i$ ), then

$$
\left(A_{\mu^{\prime}}^{\lambda}\right)^{*}=A_{\mu^{* \prime}}^{\lambda^{*}}
$$

This is a general notation which we will employ in the case of any affinor (also for the connection (2.15)).

We next consider a function of position in $X_{n}, \theta\left(\xi^{\lambda}, \xi^{\lambda^{*}}\right)$, which is analytic $\left(^{5}\right)$ in $\xi^{\lambda}, \xi^{\lambda^{*}}$ considered as independent variables. Such a function is called "semi-analytic." On the other hand, an analytic function $\phi\left(\xi^{\lambda}\right)$ of $\xi^{\lambda}$ alone is called "analytic"; all the functions studied in this paper will be assumed to be "semi-analytic" or "analytic." The question arises as to the value of $\partial \xi^{\lambda^{*}} / \partial \xi^{\lambda}$. As is easily seen, such a derivative depends on the ratio $d y^{\lambda} / d x^{\lambda}$. In order to eliminate such a possibility, it will be desirable to consider $\xi^{\lambda^{*}}, \xi^{\lambda}$ as independent variables, i.e.,

$$
\partial_{\mu} \xi^{\lambda^{*}}=0, \quad \partial_{\mu^{*}} \xi^{\lambda}=0 .
$$

We now formulate a definition of the operators $\partial_{\mu}, \partial_{\mu^{*}}$ which will check with (2.9) and (2.4) and which will replace any previous conceptions of these operators.

$$
\frac{\partial \phi}{\partial x^{\mu}}=\frac{\partial \phi}{\partial \xi^{\alpha}} \frac{\partial \xi^{\alpha}}{\partial x^{\mu}}=\partial_{\mu} \phi ; \quad \frac{\partial \phi}{\partial y^{\mu}}=\frac{\partial \phi}{\partial \xi^{\alpha}} \frac{\partial \xi^{\alpha}}{\partial y^{\mu}}=i \partial_{\mu} \phi .
$$

By multiplying the second equation by $(-i)$ and adding these two equations, the first equation of (2.4) is obtained. See Über unitäre Geometrie by J. A. Schouten und D. van Dantzig, Mathematische Annalen, vol. 103 (1930), p. 324, footnote.

(4) I, p. 22.

(5) On the elementary solution ..., T. Y. Thomas and E. W. Titt, Journal de Mathématique, vol. 18 (1939), p. 218 for definition of analytic "real" function. We assume a corresponding definition for analytic "complex" function. 
Fundamental definition. For any "semi-analytic" function $\theta$ (and for the "analytic" functions $\phi, \psi)$, the operators $\partial_{\mu}, \partial_{\mu^{*}}$ will be defined by

$$
\begin{aligned}
\partial_{\mu} \theta & =\frac{1}{2}\left(\partial \theta / \partial x^{\mu}-i \partial \theta / \partial y^{\mu}\right), \\
\partial_{\mu} \theta & =\frac{1}{2}\left(\partial \theta / \partial x^{\mu}+i \partial \theta / \partial y^{\mu}\right) .
\end{aligned}
$$

Replacing $\theta$ by $\xi^{\lambda^{*}}$ in (2.10a) and using (2.2), we see that the first equality of (2.9) is valid. The second equality of (2.9) is obtained by replacing $\theta$ in $(2.10 \mathrm{~b})$ by $\xi^{\lambda}$. Hence the definitions $(2.10)$ are consistent with the equations (2.9). Thus $\xi^{\lambda}, \xi^{\lambda^{*}}$ are independent variables with respect to differentiation. We note that $\partial\left(\xi^{\lambda^{*}}\right)^{n} / \partial \xi^{\lambda}=0$, identically in $x^{\lambda}, y^{\lambda}$.

One further question remains to be answered. "Is (2.10) identically satisfied in $x^{\lambda}, y^{\lambda}$ ?" The answer is in the affirmative. We give a proof for a semianalytic function of one complex variable and its conjugate $\theta\left(z, z^{*}\right)$. Since $\theta$ is semi-analytic, we can expand $\theta$ in a convergent power series of terms $z^{n} z^{* m}$. Applying the operator $\partial_{z}$ to this term, we obtain

$$
\partial\left(z^{n} z^{* m}\right) / \partial z=n z^{n-1} z^{* m} .
$$

Now if the expression $z^{n} z^{* m}$ is written as $f(x, y) g(x, y)$ and substituted in to the right-hand side of $(2.10 \mathrm{a})$, we obtain

$$
(g / 2)(\partial f / \partial x-i \partial f / \partial y)+(f / 2)(\partial g / \partial x-i \partial g / \partial y) .
$$

From our observation at the conclusion of the preceding paragraph, we see that the second set of terms in $(2.10 \mathrm{~d})$ vanishes. Furthermore, $(2.10 \mathrm{a})$ reduces to (2.4) (an identity) when $\phi=f=z^{n}$. That is,

$$
\frac{1}{2}(\partial f / \partial x-i \partial f / \partial y)=n z^{n-1} \text {. }
$$

Hence $(2.10 \mathrm{c})$ and $(2.10 \mathrm{~d})$ coincide and $(2.10 \mathrm{a})$ is an identity in $x, y$. The argument can be generalized to any number of variables.

We can now see why an analytic function $\phi\left(\xi^{\lambda}\right)$ of $\xi^{\lambda}$ alone is called "analytic." From (2.9), we have

$$
\partial_{\mu^{*}} \phi=0 .
$$

But upon expanding $\phi$ in to real and imaginary parts $(\phi=u+i v)$ and using the right-hand side of $(2.10 \mathrm{~b})$, we find from (2.11)

$$
\partial u / \partial x^{\mu}-\partial v / \partial y^{\mu}=0, \quad \partial v / \partial x^{\mu}+\partial u / \partial y^{\mu}=0 .
$$

These are the generalized Cauchy-Riemann equations. The conjugate equation to $(2.11)$ is

$$
\partial_{\mu} \phi^{*}=0 .
$$

Evidently, any equation implies a conjugate equation. In the future, we shall indicate the validity of the conjugate by the abbreviation "conj." It is to 
be noted, on the formal side, that in passing to the conjugate, indices are starred. The star of a starred quantity removes the original star.

We now introduce the vector $v^{\lambda}\left(\xi^{\lambda}, \xi^{\lambda^{*}}\right)$ of the first type with the transformation law

$$
v^{\lambda^{\prime}}=A_{\lambda}^{\lambda^{\prime} \nu^{\lambda}}
$$

Associated with each such vector is its conjugate, or vector of the second type $v^{\lambda^{*}}$, with transformation law

$$
v^{\lambda^{* \prime \prime}}=A_{\lambda^{*} v^{\lambda^{*}}}^{\lambda^{*}} \text {. }
$$

The theory can be extended to affinors of any mixed valence.

Let us introduce a connection in $X_{n}$ by means of the $n^{3}$ quantities $\Gamma_{\mu \alpha}^{\lambda}$ which are functions of position. Then, we define the covariant differential of a contravariant vector by

$$
\delta v^{\lambda}=d v^{\lambda}+\Gamma_{\mu \alpha}^{\lambda} v^{\alpha} d \xi^{\mu} \text {, conj. }
$$

The $\Gamma_{\mu \alpha}^{\lambda}$ transform in a well known manner( $\left.{ }^{6}\right)$ under (2.5). We now write for a covariant vector

$$
\delta w_{\lambda}=d w_{\lambda}-\Gamma_{\mu \lambda}^{\alpha} w_{\alpha} d \xi^{\mu}, \text { conj. }
$$

By expanding the ordinary differential of a vector, we obtain

$$
d v^{\lambda}=d \xi^{\mu} \partial_{\mu} v^{\lambda}+d \xi^{\mu} \partial_{\mu} v^{\lambda}, \text { conj. }
$$

If we define the covariant derivative of $w_{\lambda}, v^{\lambda}$ by means of

$$
\begin{aligned}
\nabla_{\mu} v^{\lambda} & =\partial_{\mu} v^{\lambda}+\Gamma_{\mu \alpha}^{\lambda} v^{\alpha}, & \nabla_{\mu} w_{\lambda} & =\partial_{\mu} w_{\lambda}-\Gamma_{\mu \lambda}^{\alpha} w_{\alpha}, \\
\nabla_{\mu * v}{ }^{\lambda} & =\partial_{\mu * v}{ }^{\lambda}, & \nabla_{\mu *} w_{\lambda} & =\partial_{\mu^{*}} w_{\lambda}, \text { conj. },
\end{aligned}
$$

then

$$
\begin{aligned}
\delta v^{\lambda} & =d \xi^{\mu} \nabla_{\mu} v^{\lambda}+d \xi^{\mu^{*}} \nabla_{\mu^{*}} v^{\lambda}, \text { conj., } \\
\delta w_{\lambda} & =d \xi^{\mu} \nabla_{\mu} w_{\lambda}+d \xi^{\mu^{*}} \nabla_{\mu^{*}} w_{\lambda}, \text { conj. }
\end{aligned}
$$

An hermitian $X_{n}$ with a covariant derivative defined by $(2.18),(2.19)$ is denoted by $K_{n}$.

We now introduce an hermitian tensor with hermitian symmetry

$$
a_{\lambda \mu^{*}}=\left(a_{\lambda \mu^{*}}\right)^{* \prime}=a_{\mu^{*} \lambda},
$$

the $(')$ indicating the transpose matrix. If we condition $a_{\lambda \mu^{*}}$ by requiring that

(6) I, p. 75, (7.4). 


$$
\delta a_{\lambda \mu^{*}}=0=\left(\partial_{\nu} a_{\lambda \mu^{*}}-\Gamma_{\nu \lambda}^{\rho} a_{\rho \mu^{*}}\right) d \xi^{\nu}+\left(\partial_{\nu^{*}} a_{\lambda \mu^{*}}-\Gamma_{\nu^{*} \mu^{*}}^{\rho^{*}} a_{\lambda \rho^{*}}\right) d \xi^{\nu^{*}},
$$

then the space $K_{n}$ becomes a unitary $K_{n}$. For such a space, from (2.23), we can prove $\left({ }^{7}\right)$

$$
\begin{aligned}
\nabla_{\nu} a_{\lambda \mu^{*}} & =\partial_{\nu} a_{\lambda \mu^{*}}-\Gamma_{\nu \lambda}^{\rho} a_{\rho \mu^{*}}=0, \text { conj., } \\
\nabla_{\nu^{*}} a_{\lambda \mu^{*}} & =\partial_{\nu^{*}} a_{\lambda \mu^{*}}-\Gamma_{\nu^{*} \mu^{*}}^{\rho^{*}} a_{\lambda \rho^{*}}=0, \text { conj. }
\end{aligned}
$$

The $a_{\lambda \mu^{*}}$ is now a fundamental tensor and can be used to raise and lower indices through the $\nabla$ operator; that is, if

$$
v_{\lambda}=a_{\lambda \mu * \nu^{* *}} \text {, conj., }
$$

then

$$
\nabla_{\alpha} v_{\lambda}=a_{\lambda \mu^{*}} \nabla_{\alpha} v^{\mu^{*}}, \text { conj. }
$$

If we define the contravariant fundamental tensor $a^{\sigma^{*} \kappa}$ by

$$
a^{\sigma^{*} \kappa} a_{\lambda \sigma^{*}}=A_{\lambda}^{\kappa}, \text { conj., }
$$

then (2.24), (2.25) may be solved for the connection

$$
\begin{aligned}
\Gamma_{\mu \lambda}^{\alpha} & =\left(\partial_{\mu} a_{\lambda \sigma^{*}}\right) a^{\sigma^{* \alpha}}=\left(\partial_{\mu} a_{\sigma^{*} \lambda}\right) a^{\alpha \sigma^{*}}, \\
\Gamma_{\mu^{*} \lambda^{*}}^{\kappa^{*}} & =\left(\partial_{\mu^{*}} a_{\sigma \lambda^{*}}\right) a^{\kappa^{*} \sigma}=\left(\partial_{\mu^{*}} a_{\lambda^{*} \sigma}\right) a^{\sigma \alpha^{*}} .
\end{aligned}
$$

For the curvature affinor, we have the components $\left({ }^{8}\right)$,

$$
\begin{aligned}
& R_{\nu \mu \lambda}^{\cdots \kappa}=2 \partial_{[\nu} \Gamma_{\mu] \lambda}^{\kappa}+2 \Gamma_{[\nu|\rho|}^{\kappa} \Gamma_{\mu] \lambda}^{\rho}, \text { conj. }, \\
& R_{\nu^{*} \mu \lambda}^{\cdots \kappa}=\partial_{\nu^{*}} \Gamma_{\mu \lambda}{ }^{\kappa}, \text { conj. }
\end{aligned}
$$

The sign [ ] on the indices means that the alternating product is to be formed; the sign ( ) enclosing indices means that the symmetric product is to be formed; the sign || implies that indices enclosed are not to be included in any alternating or symmetric product $\left({ }^{9}\right)$.

For unitary $K_{n}$, we find from $(2.29)\left({ }^{10}\right)$

$$
R_{\nu \mu \lambda}^{\cdots \times}=0 \text {, conj. }
$$

Next, we introduce the torsion affinor

$$
S_{\mu \lambda}^{\cdot \cdot \kappa}=\Gamma_{[\mu \lambda]}^{\kappa} \text {, conj. }
$$
(7) II, p. 234, (21.40).
(8) II, p. 233, (21.33).
(श) I, pp. 14, 15.
(10) II, p. 235, (21.43). 
If this affinor does not vanish in $K_{n}$, then we may say that $K_{n}$ has torsion.

Finally, we introduce an unitary-orthogonal ennuple( $\left.{ }^{11}\right)$ at each point $P$ of $K_{n}$. We may denote these vectors by $u^{\lambda}\left(\xi^{\lambda}, \xi^{\lambda^{*}}\right)$ (subindex $k=1, \cdots, n$ ) where

$$
a_{\lambda \alpha^{*}} u_{k}^{\lambda} u_{j}^{\alpha^{*}}=\underset{k j}{\delta} ; \quad u_{k}^{\lambda} u_{\lambda}^{j}=\oint_{k}^{j}, \text { conj. }
$$

Here $\delta$ is the Kronecker symbol. We note that at each point $P$ there are $\infty^{n(n-1) / 2}$ such ennuples. Let ' $K_{n}$ be another unitary space also mapped by the variables $\xi^{\lambda}$. We say that the point $P\left(\xi^{\lambda}\right)$ of $K_{n}$ corresponds to the point ${ }^{\prime} P\left(\xi^{\lambda}\right)$ of ' $K_{n}$ and conversely. Let ' $u^{\lambda}\left(\xi^{\lambda}, \xi^{\lambda^{*}}\right)$, subindex $k=1, \cdots, n$, denote an unitary orthogonal ennuple at ${ }^{\prime} P\left(\xi^{\lambda}\right)$. We now form the definitions

Definition 1. We shall say that two ennuples of $K_{n}$ and ' $K_{n}$ at corresponding points $P$ and 'P are corresponding ennuples if functions $\alpha\left(\xi^{\lambda}, \xi^{\lambda^{*}}\right)$, subindex $j=1, \cdots, n$, exist so that

$$
{ }_{j}^{\prime} u_{\lambda}=\underset{j}{\alpha}{ }_{j}^{\prime} u_{\lambda}, \quad \text { not summed on } j \text {. }
$$

Definition 2. If the $\alpha$ (subindex $j$ ) are all equal, we say that the two ennuples correspond conformally.

III. Conformal unitary spaces; correspondence of paths. Consider two unitary spaces $K_{n},{ }^{\prime} K_{n}$ both mapped by the variables $\xi^{\lambda}$ and whose fundamental tensors are related by

$$
{ }^{\prime} a_{\lambda \mu^{*}}=\sigma a_{\lambda \mu^{*}}, \text { conj. }
$$

Here $\sigma$ is a function of $\xi^{\lambda}, \xi^{\lambda^{*}}$. Taking the conjugate of (3.1) and using (2.22), we see that $\sigma$ is real. Hence,

Definition 3. Two unitary spaces whose fundamental tensors are related by (3.1) where $\sigma$ is real will be said to be conformal.

By use of (3.1) and (2.29), we find

$$
\Gamma_{\mu \lambda}^{\kappa}=\Gamma_{\mu \lambda}^{\kappa}+A_{\lambda}^{\kappa} \partial_{\mu} \log \sigma, \text { conj. }
$$

We shall now prove the theorem

THEOREM 1. Unitary spaces $K_{n},{ }^{\prime} K_{n}$, both without torsion, cannot be conformal, unless $K_{n}$ and ' $K_{n}$ are the same space.

For if these spaces were conformal, then (3.2) would be valid. By our assumption, the connections of both spaces are symmetric. Thus

(11) I, p. 235. 


$$
A_{[\mu}^{\kappa} \partial_{\lambda]} \log \sigma=0, \text { conj. }
$$

By expanding, we find

$$
\partial_{\mu} \log \sigma=0, \text { conj. }
$$

Hence $\sigma$ is a constant. Such a conformal transformation takes $K_{n}$ into itself.

We shall assume in the future that at least one of the unitary spaces $K_{n}$, ' $K_{n}$ has torsion. If only one of these spaces has torsion $\left(K_{n}\right)$, then from (2.35), (3.2) we see that

$$
\stackrel{S_{\mu \lambda}^{* \kappa}}{*}=-A_{[\mu}^{\kappa} p_{\lambda]}, \quad p_{\lambda}=\partial_{\lambda} \log \sigma, \text { conj. }
$$

The connection of $K_{n}$ is then said to be semi-symmetric $\left({ }^{12}\right)$. In the general case, we may write (3.2) in the form

$$
{ }^{\prime} \Gamma_{(\mu \lambda)}^{\kappa}=\Gamma_{(\mu \lambda)}^{\kappa}+A_{(\mu}^{\kappa} p_{\lambda)}, \text { conj. }
$$

If we denote these symmetric parts of the connection by

$$
\prime^{\delta}{ }_{\mu \lambda}^{\kappa}={ }^{\prime} \Gamma_{(\mu \lambda)}^{\kappa}, \quad \stackrel{8}{\Gamma}_{\mu \lambda}^{\kappa}=\Gamma_{(\mu \lambda)}^{\kappa}, \text { conj. }
$$

and replace the vector $p_{\lambda}$ by

$$
q_{\lambda}=p_{\lambda} / 2=\partial_{\lambda} \log \sigma / 2, \text { conj.; }
$$

then (3.6) becomes

$$
\dot{\Gamma}_{\mu \lambda}^{\kappa}=\stackrel{\delta}{\Gamma}_{\mu \lambda}^{\kappa}+2 A_{(\mu}^{\kappa} q_{\lambda)}, \text { conj. }
$$

But, the equations (3.9) are well known in the theory of paths. These equations state that the paths of $K_{n}$ and ' $K_{n}$ correspond( $\left.{ }^{13}\right)$. Hence, we have the theorem:

THEOREM 2. If two unitary spaces are conformal, then their paths correspond.

This last theorem has no equivalent in the theory of real spaces.

We shall now prove a converse of Theorem 2. Consider two unitary spaces: ' $K_{n}$ without torsion; $K_{n}$ with torsion. Let us assume that the paths of these spaces correspond. Hence the connections of these spaces are related by

$$
\Gamma_{\mu \lambda}^{\kappa}=\stackrel{8}{\Gamma}_{\mu \lambda}^{\kappa}+2 A_{(\mu}^{\kappa} q_{\lambda)}, \text { conj. }
$$

where

$$
\stackrel{8}{\Gamma}_{\mu \lambda}^{\kappa}=\Gamma_{(\mu \lambda)}^{\kappa}, \text { conj. }
$$

(12) I, p. 82, (7.27).

(19) II, p. 176, (18.1). 
In our work, we shall find it useful to consider the particular correspondence of paths for which $q_{\lambda}\left(\xi^{\lambda}, \xi^{\lambda^{*}}\right)$ is a gradient vector. Hence, we define:

DEFINITION 4. The particular correspondence of paths determined by (3.10) when $q_{\lambda}$ is a gradient vector of a real scalar function of position shall be called restricted correspondence of paths.

The curvature affinors determined by the connections occurring in (3.10) may be found from (2.31), (2.32). With the aid of (3.10), we find that these affinors are related by $\left({ }^{14}\right)$

$$
\begin{aligned}
& { }^{\prime} R_{\nu \mu \lambda}^{\cdots \kappa}=\stackrel{s}{R}_{\nu \mu \lambda}^{\cdots \kappa}+2 A_{[\nu}^{\kappa} q_{\mu] \lambda}-2 q_{[\nu \mu]} A_{\lambda}^{\kappa}, \text { conj., } \\
& { }^{\prime} R_{\nu^{*} \mu \lambda}^{\cdots \kappa}=\stackrel{s}{R}_{\nu^{*} \mu \lambda}^{\cdots \kappa}+2\left(\dot{\nabla}_{\nu^{*}}^{*} q_{(\mu}\right) A_{\lambda)}^{\kappa}, \text { conj., }
\end{aligned}
$$

where

$$
q_{\nu \mu}=-\dot{\nabla}_{\nu} q_{\mu}+q_{\nu} q_{\mu}, \text { conj. }
$$

In these equations, the $\nabla_{\mu}$ (superindex $s$ ) represents covariant differentiation with respect to the connection occurring in the right-hand side of (3.10). Furthermore, by (2.33), the left-hand side of (3.12) vanishes. Finally, we define the affinors

$$
\begin{aligned}
& { }^{\prime} R_{\alpha \lambda^{*} \cdot \nu}^{\cdots \beta}={ }^{\cdots} R_{\alpha \mu^{*} \lambda^{*}}^{\cdots \alpha^{*}} a^{\lambda * \beta}{ }^{\lambda{ }^{*}} a_{\nu \kappa^{*}}, \text { conj., } \\
& { }^{\prime} C_{\alpha \mu^{*} \nu}^{\cdots \beta}={ }^{\prime} R_{\alpha \mu^{*} \cdot \nu}^{\cdots \beta}-{ }^{\prime} R_{\mu^{*} \alpha \nu}^{\cdots \beta}, \text { conj. }
\end{aligned}
$$

We may now state our theorem as:

THEOREM 3. If two unitary spaces, ' $K_{n}$ without torsion, $K_{n}$ with torsion are given, and if (1) the paths of these spaces are in restricted correspondence, (2) the affinor ' $C_{\alpha \mu^{\prime} \nu}^{*}$ vanishes, (3) one arbitrary unitary orthogonal ennuple at one arbitrary point $P$ of $K_{n}$ is conformal to the corresponding ennuple at the corresponding point ' $P$ of ' $K_{n}$ (see (2.36)), then the two spaces are conformal.

By assumption (1), the equation (3.10) is valid. Substituting (2.29) into (3.10) and transvecting with ' $a_{\kappa \alpha^{*}}$, we find

$$
\partial_{\mu}{ }^{\prime} a_{\lambda \alpha^{*}}=\dot{\delta}_{\Gamma \lambda}^{\kappa}{ }^{\prime} a_{\kappa \alpha^{*}}+{ }^{\prime} a_{\mu \alpha^{*}} q_{\lambda}+{ }^{\prime} a_{\lambda \alpha^{*}} q_{\mu}, \text { conj. }
$$

We shall consider (3.17) as a system of partial differential equations in ' $a_{\lambda \alpha^{*}}$ where $\Gamma_{\mu \lambda}^{\kappa}$ (superindex $s$ ) and $q_{\lambda}$ are considered as known. The integrability conditions of (3.17) are

$$
\begin{aligned}
\partial_{[\nu} \partial_{\mu]^{\prime}} a_{\lambda \alpha^{*}} & =0, \text { conj., } \\
\partial_{\left[\nu^{*}\right.} \partial_{\mu]^{\prime}} a_{\lambda \alpha^{*}} & =0, \text { conj. }
\end{aligned}
$$

(14) II, p. 240, (21.62). 
Our method of procedure is to show that because of our assumptions, the equations (3.18), (3.19) are identically satisfied. It then follows that there exists an unique solution for ' $a_{\lambda \mu^{*}}$ which by $(2.22)$ contains $n(n+1) / 2$ constants of integration. From (3.11), (2.29) the equation (3.17) may be written in the form

$$
\partial_{(\mu}{ }^{\prime} a_{\lambda) \alpha^{*}}=\left(\partial_{(\mu} a_{\lambda) \beta^{*}}\right) a^{\beta^{* \kappa}}{ }^{\prime} a_{\kappa \alpha^{*}}+{ }^{\prime} a_{\mu \alpha^{*}} q_{\lambda}+{ }^{\prime} a_{\lambda \alpha^{*}} q_{\mu} \text {, conj. }
$$

Since the connection of ' $K_{n}$ is symmetric, (3.20) may also be written in the form

$$
\partial_{\mu}^{\prime} a_{\lambda \alpha^{*}}=\left(\partial_{(\mu} a_{\lambda) \beta^{*}}\right) a^{\beta^{* \kappa}}{ }^{\prime} a_{\kappa \alpha^{*}}+{ }^{\prime} a_{\mu \alpha^{*}} q_{\lambda}+{ }^{\prime} a_{\lambda \alpha^{*}} q_{\mu} \text {, conj. }
$$

This last set of equations constitutes a system of linear homogeneous partial differential equations. For, we can write $(3.20 \mathrm{~b})$ in the form

$$
\partial_{\mu}{ }^{\prime} a_{\lambda \alpha^{*}}-\stackrel{A_{\mu \lambda}^{* \nu}}{\prime \prime} a_{\nu \alpha^{*}}=0, \text { conj., }
$$

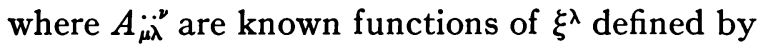

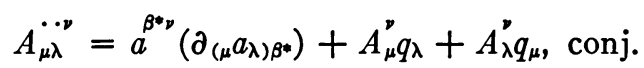

If $n(n+1) / 2$ particular integrals of (3.20c) are denoted by

$$
{ }^{\prime}{ }_{a \lambda \alpha^{*}}^{k}, \quad k=1, \cdots, n(n+1) / 2,
$$

then the general solution of $(3.20 \mathrm{c})$ is given by

$$
' a_{\lambda \alpha^{*}}=c_{k}^{\prime}{ }^{\prime k} a_{\lambda \alpha^{*}}, \quad k=1, \cdots, n(n+1) / 2 ; \text { conj.; }
$$

where $c$, subindex $k$, are arbitrary complex constants. By substitution, we see that one particular solution of (3.20a) or (3.20c) is

$$
{ }^{\prime} a_{\lambda \alpha^{*}}=\sigma a_{\lambda \alpha^{*}}, \text { conj., }
$$

where $\sigma$ is a real solution of

$$
\partial_{\mu} \log \sigma=2 q_{\mu}, \text { conj. }
$$

Since $q_{\mu}$ is the gradient of a real scalar $\phi$, the solution of (3.21d) for $\sigma$ is given by

$$
\log \sigma=2 \phi+f\left(\xi^{\lambda *}\right)+s,
$$

where $s$ is an arbitrary constant and $f$ is an arbitrary function. The conjugate equation of (3.21d) yields

$$
\log \sigma=2 \phi+g\left(\xi^{\lambda}\right)+r .
$$

This last result follows from the facts that $\sigma, \phi$ are real; $r$ is an arbitrary con- 
stant and $g$ is an arbitrary function. Upon taking the conjugate of (3.21e), we find

$$
s^{*}+f^{*}\left(\xi^{\lambda}\right)=g^{*}\left(\xi^{\lambda^{*}}\right)+r^{*} .
$$

Since $\xi^{\lambda}, \xi^{\lambda^{*}}$ are independent, the functions $f, g$ both vanish. Furthermore, $s=r$ and $s$ is real. Thus $\sigma$ is given by

$$
\sigma=S e^{2 \phi}
$$

real $S$ is a real arbitrary constant.

The general solution of (3.20c) can now be written as

$$
{ }^{\prime} a_{\lambda \alpha^{*}}=\sigma a_{\lambda \alpha^{*}}+c_{k}^{\prime}{ }^{\prime k} a_{\lambda \alpha^{*}}, \quad k=2, \cdots, n(n+1) / 2 ; \text { conj. }
$$

Note, that $\sigma$ contains an arbitrary constant. We now use condition (3) of our theorem to show that $c$ (subindex $k=2, \cdots$ ) must be zero. At $P$, we expand ' $a_{\lambda \alpha^{*}}$ (superindex $k$ ) in terms of the ennuple and find

$$
\prime_{a_{\lambda \alpha^{*}}}^{k}=\underset{j l}{k} \stackrel{i}{i} \stackrel{u}{\lambda}_{\lambda}^{l} u_{\alpha^{*}}, \text { conj. }
$$

By substituting (3.22b) into (3.22a) and transvecting with the $u_{\lambda}, u_{\lambda^{*}}$ vectors, we obtain by use of (2.35) and (2.36) - where all $\alpha$ 's are equal-

$$
\begin{array}{ll}
\underset{k}{c \underset{j l}{a}=0,} & j \neq l, j, l=1, \cdots, n, \\
\underset{k}{c \underset{j j}{a}=\left(\alpha \alpha^{*}-\sigma\right),} & k=2, \cdots, n(n+1) / 2 .
\end{array}
$$

We can evidently fix the arbitrary constant in $\sigma$ so that the right-hand side of (3.22d) vanishes at some point $P$. Let us assume that the $c$ (subindex $k$ ) are not all zero. Then the $[n(n+1) / 2-1]$ solutions ' $a_{\lambda \mu^{*}}$ (superindex $k$ ) are linearly related at $P$. Since the ' $a_{\lambda \mu^{*}}$ (superindex $k$ ) satisfy a system of linear partial differential equations, these ' $a_{\lambda \mu^{*}}$ are not independent in $K_{n}$. It will be shown in (3.41) that the in tegrability conditions of (3.20a) are identically satisfied in virtue of condition (2) of our theorem. Hence, from the theory of linear differential equations, (3.20a) has $n(n+1) / 2$ independent integrals $\sigma a_{\lambda \mu^{*}},{ }^{\prime} a_{\lambda \mu^{*}}$ (superindex $k$ ). Thus $c$ (subindex $k$ ) must vanish and the only solution of (3.20a) under the condition (3) of our theorem is

$$
{ }^{\prime} a_{\lambda \alpha^{*}}=\sigma a_{\lambda \alpha^{*}} \text {, conj. }
$$

Thus, we have reduced our problem to showing that the conditions (3.18), (3.19) are identically satisfied. We first consider (3.18). From (3.17), we find

$$
\begin{aligned}
& \partial_{[\nu} \partial_{\mu]}{ }^{\prime} a_{\lambda \alpha^{*}}={ }^{\prime} a_{\kappa \alpha^{*}}\left(\partial_{[\nu} \stackrel{8}{\Gamma}_{\mu] \lambda}^{\kappa}\right)+\dot{\Gamma}_{\lambda[\mu}^{\kappa} \partial_{\nu]}{ }^{\prime} a_{\kappa \alpha^{*}}+q_{\lambda} \partial_{[\nu}{ }^{\prime} a_{\mu] \alpha^{*}} \\
& +q_{[\mu} \partial_{\nu]}{ }^{\prime} a_{\lambda \alpha^{*}}+\left(\partial_{[\nu} q_{|\lambda|}\right)^{\prime} a_{\mu] \alpha^{*}}+{ }^{\prime} a_{\lambda \alpha^{*}} \partial_{[\nu} q_{\mu]} \text {, conj. }
\end{aligned}
$$


With the aid of (3.17), we see that the third term on the right-hand side of (3.24) vanishes. We next simplify terms two and four:

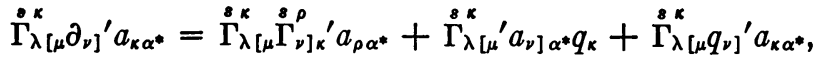

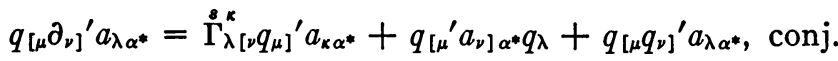

Upon substituting these simplifications into (3.24) and using (2.31), (3.14), we obtain

$$
\partial_{[\nu} \partial_{\mu]}{ }^{\prime} a_{\lambda \alpha^{*}}=\frac{1}{2} \dot{R}_{\nu \mu \lambda}^{\cdots \kappa}{ }^{\cdots} a_{\kappa \alpha^{*}}+q_{\lambda[\mu}{ }^{\prime} a_{\nu] \alpha^{*}}-q_{[\nu \mu]}{ }^{\prime} a_{\lambda \alpha^{*}}, \text { conj. }
$$

From (3.12), (3.27) we find

$$
2^{\prime} a^{\alpha * \beta} \partial_{[\nu} \partial_{\mu]}{ }^{\prime} a_{\lambda \alpha^{*}}={ }^{\prime} R_{\nu \mu \lambda}^{\cdots \beta} \text {, conj. }
$$

Since the right-hand side of (3.28) vanishes for a unitary ' $K_{n}$, we see that the conditions (3.18) are identically satisfied.

The verification of (3.19) requires considerable computation due to the unsymmetric character of the indices. We shall list the principal steps. By taking the conjugate of (3.9) and using (2.30), we find

$$
\partial_{\mu^{*}} a_{\nu \lambda^{*}}=\stackrel{8}{\Gamma}_{\mu^{* * \lambda^{*}}}^{\kappa^{*}} a_{\nu \kappa^{*}}+{ }^{\prime} a_{\nu \mu^{*}} q_{\lambda^{*}}+{ }^{\prime} a_{\nu \lambda^{*}} q_{\mu^{*}} \text {, conj. }
$$

By differentiation of (3.29),

$$
\begin{aligned}
\partial_{\alpha} \partial_{\mu^{*}} a_{\nu \lambda^{*}}= & \left(\partial_{\alpha} \Gamma_{\mu^{*} \lambda^{*}}^{\alpha^{*}}\right)^{\prime} a_{\nu \kappa^{*}}+\left(\partial_{\alpha}^{\prime} a_{\nu \nu^{*}}\right){\stackrel{\rho}{\Gamma_{\mu^{*} \lambda^{*}}}}^{\kappa^{*}}+\left(\partial_{\alpha^{\prime}}^{\prime} a_{\nu \mu^{*}}\right) q_{\lambda^{*}} \\
& +{ }^{\prime} a_{\nu \mu^{*}} \partial_{\alpha} q_{\lambda^{*}}+\left(\partial_{\alpha}^{\prime} a_{\nu \lambda^{*}}\right) q_{\mu^{*}}+{ }^{\prime} a_{\nu \lambda^{*}} \partial_{\alpha} q_{\mu^{*}}, \text { conj. }
\end{aligned}
$$

We simplify terms two, three and five of the right-hand side by means of

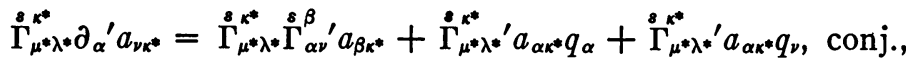

$$
\begin{aligned}
& \left(\partial_{\alpha}^{\prime} a_{\nu \mu^{*}}\right) q_{\lambda^{*}}=\dot{\Gamma}_{\alpha \nu}^{\prime \alpha} a_{\kappa \mu^{*}} q_{\lambda^{*}}+{ }^{\prime} a_{\alpha \mu^{*}} q_{\nu} q_{\lambda^{*}}+{ }^{\prime} a_{\nu \mu^{*}} q_{\alpha} q_{\lambda^{*}} \text {, conj., } \\
& \left(\partial_{\alpha}^{\prime} a_{\nu \lambda^{*}}\right) q_{\mu^{*}}=\stackrel{8}{\Gamma}_{\alpha \nu}^{\alpha} a_{\kappa \lambda^{*}} q_{\mu^{*}}+{ }^{\prime} a_{\alpha \lambda^{*}} q_{\nu} q_{\mu^{*}}+{ }^{\prime} a_{\nu \lambda^{*}} q_{\alpha} q_{\mu^{*}} \text {, conj. }
\end{aligned}
$$

From (3.17), by differentiation, we get

$$
\begin{aligned}
& \partial_{\mu^{*}} \partial_{\alpha}{ }^{\prime} a_{\nu \lambda^{*}}=\left(\partial_{\mu^{*}} \stackrel{\delta}{\Gamma}_{\alpha \nu}^{\alpha}\right)^{\prime} a_{\kappa \lambda^{*}}+\dot{\Gamma}_{\alpha \nu}^{\kappa} \partial_{\mu^{*}} a_{\kappa \lambda^{*}}+\left(\partial_{\mu^{*}}{ }^{\prime} a_{\alpha \lambda^{*}}\right) q_{\nu} \\
& +{ }^{\prime} a_{\alpha \lambda^{*}} \partial_{\mu^{*}} q_{\nu}+\left(\partial_{\mu^{*}}{ }^{\prime} a_{\nu \lambda^{*}}\right) q_{\alpha}+{ }^{\prime} a_{\nu \lambda^{*}} \partial_{\mu^{*}} q_{\alpha} \text {, conj. }
\end{aligned}
$$

For the terms two, three and five of (3.34), we obtain

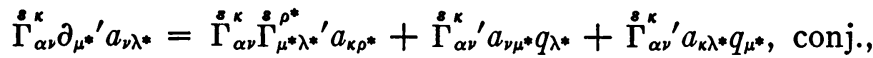

$$
\begin{aligned}
& \left(\partial_{\mu^{*}} a_{\alpha \lambda^{*}}\right) q_{\nu}=\dot{\Gamma}_{\mu^{*} \lambda^{*}}^{\kappa^{*}} a_{\alpha \kappa^{*}} q_{\nu}+{ }^{\prime} a_{\alpha \mu^{*}} q_{\lambda^{*}} q_{\nu}+{ }^{\prime} a_{\alpha \lambda^{*}} q_{\mu^{*}} q_{\nu} \text {, conj., } \\
& \left(\partial_{\mu^{*}}{ }^{\prime} a_{\nu \lambda^{*}}\right) q_{\alpha}={\stackrel{8}{\Gamma^{\prime *}} \mu^{*} \lambda^{*}}^{\prime} a_{\nu \lambda^{*}} q_{\alpha}+{ }^{\prime} a_{\nu \lambda^{*}} q_{\mu^{*}} q_{\alpha}+{ }^{\prime} a_{\nu \mu^{*}} q_{\lambda^{*}} q_{\alpha} \text {, conj. }
\end{aligned}
$$


When we subtract (3.30) from (3.34), we find that the set (3.31), (3.32), (3.33) cancels against (3.35), (3.36), (3.37). And we obtain

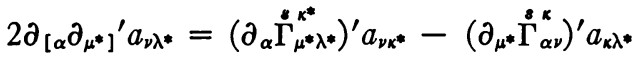

$$
\begin{aligned}
& +{ }^{\prime} a_{\nu \mu^{*}} \partial_{\alpha} q_{\lambda^{*}}+{ }^{\prime} a_{\nu \lambda^{*}} \partial_{\alpha} q_{\mu^{*}}-{ }^{\prime} a_{\alpha \lambda^{*}} \partial_{\mu^{*}} q_{\nu}-{ }^{\prime} a_{\nu \lambda^{*}} \partial_{\mu^{*}} q_{\alpha} \text {, conj. }
\end{aligned}
$$

This equation may be simplified by transvection and use of (2.32) into

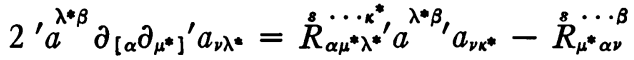

$$
\begin{aligned}
& +{ }^{\prime}{ }^{\lambda * \beta}{ }^{\prime} a_{\nu \mu^{*}} \partial_{\alpha} q_{\lambda^{*}}+A_{\nu}^{\beta} \partial_{\alpha} q_{\mu^{*}}-A_{\alpha}^{\beta} \partial_{\mu^{*}} q_{\nu}-A_{\nu}^{\beta} \partial_{\mu^{*}} q_{\alpha} \text {, conj. }
\end{aligned}
$$

Upon replacing the first and second terms of the right-hand side by their values as determined by (3.13), and using (3.15), we find

$$
2^{\prime} a^{\lambda * \beta} \partial_{[\alpha} \partial_{\left.\mu^{*}\right]}^{\prime} a_{\nu \lambda^{*}}={ }^{\prime} R_{\alpha \mu^{*} \cdot \nu}^{\cdot \beta}-{ }^{\cdots \cdot} R_{\mu^{*} \alpha \nu}^{\cdots \beta} \text {, conj. }
$$

From (3.40), (3.16), these last integrability conditions are equivalent to

$$
{ }^{\prime} C_{\alpha \mu^{*} \nu}^{\cdots \beta}=0, \text { conj. }
$$

Hence our theorem is proved.

The extension of this theorem to $K_{n},{ }^{\prime} K_{n}$ both with torsion is not possible. For then the term ' $S_{\mu \lambda}^{\cdot *{ }^{*} \prime} a_{\kappa \alpha^{*}}$ must be added to the right-hand side of (3.17); the argument $(3.20,3.21)$ is then no longer valid.

IV. Conformal unitary $k$-spaces; a conformal invariant. We first define a unitary $k$-space by

DEFINITION 5. If the fundamental tensor of a unitary space $K_{n}$ can be written in the form $a_{\alpha \nu^{*}}=\beta \partial_{\alpha} \partial_{\nu^{*}} \log k$, where $k$ is real and $\beta$ is an arbitrary function of $\xi^{\lambda}, \xi^{\lambda^{*}}$ then $K_{n}$ shall be said to be a unitary $k$-space.

From (2.29), we see that those $k$-spaces for which $\beta=1$ have a symmetric connection. Such spaces have been studied by Mitrochin, Fuchs, Bergman and others $\left({ }^{15}\right)$. The class of $k$-spaces with real $\beta$ are evidently conformal to those $k$-spaces for which $\beta=1$ (fixed $k$ ). Hence by Theorem 1 , the connections of the class of $k$-spaces with real $\beta$ ( $\beta$ not constant) possess torsion.

If we fix the function $k$, but allow $\beta$ to vary, then we obtain a set of such $k$-spaces. In particular, we consider those $\beta$ which are the product of a real function and an initial $\beta_{1}$. Any two spaces of such a set are conformal to each other. We define a subset of such spaces by the following:

(15) Über die Veränderung der Krïmmung von Hyperflächen bei Pseudokonformen Abbildungen, by I. Mitrochin, Bulletin de l' Institute de Mathématiques et Mécanique à l' Université Koubycheff de Tomsk, vol. 1 (1937), no. 3. 
Definition 6. All unitary $k$-spaces (fixed $k$ ) whose fundamental tensors are related by

$$
{ }^{\prime} a_{\lambda \mu^{*}}=k^{\alpha} a_{\lambda \mu^{*}}, \text { conj., }
$$

where $\alpha$ is any real number, will be said to be $k$-conformal.

This definition imposes the following restrictions on $\beta$. Consider two $k$ spaces

$$
\begin{aligned}
a_{\lambda \mu^{*}} & =\beta_{1} \partial_{\lambda} \partial_{\mu^{*}} \log k, \text { conj., } \\
{ }^{\prime} a_{\lambda \mu^{*}} & =\beta_{2} \partial_{\lambda} \partial_{\mu^{*}} \log k, \text { conj. }
\end{aligned}
$$

If these $k$-spaces are $k$-conformal, then

$$
k^{\alpha} \beta_{1}=\beta_{2} .
$$

Evidently, the $\infty^{1} k$-spaces whose first fundamental tensors are

$$
\beta_{1} \partial_{\lambda} \partial_{\mu^{*}} \log k ; \quad k^{\alpha} \beta_{1} \partial_{\lambda} \partial_{\mu^{*}} \log k ; \quad k^{\alpha^{\prime}} \beta_{1} \partial_{\lambda} \partial_{\mu^{*}} \log k \text {, etc., }
$$

are $k$-conformal to each other when $\alpha, \alpha^{\prime}$, etc., are any real numbers. We now prove the theorem

THEOREM 4. The affinor $C_{\alpha \mu{ }^{*}{ }^{\circ}}^{\cdots \cdots}$ is a conformal invariant of all unitary $k$-spaces which are $k$-conformal.

From (3.16), (3.10), (2.32), we find for any two unitary conformal spaces

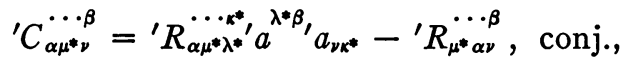

$$
\begin{aligned}
& { }^{\prime} C_{\alpha \mu^{*} \nu}^{\cdots \beta}=\left(\partial_{\alpha} \Gamma_{\mu^{*} \lambda^{*}}^{\boldsymbol{\beta}^{*}}+A_{\mu^{*}}^{\boldsymbol{\kappa}^{*}} \partial_{\alpha} q_{\lambda^{*}}+A_{\lambda^{*}}^{\kappa^{*}} \partial_{\alpha} q_{\mu^{*}}\right)^{\prime} a^{\lambda^{*} \beta} a_{\nu k^{*}} \\
& \text { - }\left(\partial_{\mu^{*}} \dot{\Gamma}_{\alpha \nu}^{\beta}+A_{\alpha}^{\beta} \partial_{\mu^{*}} q_{\nu}+A_{\nu}^{\beta} \partial_{\mu^{*}} q_{\alpha}\right) \text {, conj. }
\end{aligned}
$$

Simplifying (4.7) with the aid of (3.16), we obtain

$$
\begin{aligned}
{ }^{\prime} C_{\alpha \mu^{*} \nu}^{\cdots \beta}= & C_{\alpha \mu^{*} \nu}^{\cdots \beta}+{ }^{\cdots * \beta} a^{\lambda^{*} \beta} a_{\nu \mu^{*}} \partial_{\alpha} q_{\lambda^{*}}+A_{\nu}^{\beta} \partial_{\alpha} q_{\mu^{*}} \\
& -A_{\alpha}^{\beta} \partial_{\mu^{*}} q_{\nu}-A_{\nu}^{\beta} \partial_{\mu^{*}} q_{\alpha}, \text { conj. }
\end{aligned}
$$

Since our spaces are $k$-spaces and $k$-conformal, then (4.2), (4.3), (4.4) are valid. Furthermore, from (3.8)

$$
q_{\lambda^{*}}=\frac{1}{2} \partial_{\lambda^{*}} \log \sigma, \text { conj. }
$$

Hence from (4.1), where $\sigma=k^{\alpha}$, and (4.3), we find

$$
\partial_{\mu} q_{\lambda^{*}}=(\alpha / 2) \partial_{\mu} \partial_{\lambda^{*}} \log k=\alpha^{\prime} a_{\mu \lambda^{*}} / 2 \beta_{2}, \text { conj. }
$$

From (4.9) conj., we have

$$
\partial_{\lambda^{*}} q_{\mu}=(\alpha / 2) \partial_{\lambda^{*}} \partial_{\mu} \log k=\alpha^{\prime} a_{\mu \lambda^{*}} / 2 \beta_{2}, \text { conj. }
$$


From (4.10), (4.11), we find the important relation

$$
\partial_{\lambda^{*}} q_{\mu}=\partial_{\mu} q_{\lambda^{*}}, \text { conj. }
$$

Upon substituting (4.11), (4.12) into (4.8), we see that

$$
{ }^{\prime} C_{\alpha \mu^{* \nu}}^{\cdots \beta}=C_{\alpha \mu^{*} \nu}^{\cdots \beta} \text {, conj. }
$$

Hence our theorem is proved.

We may also prove the converse theorem:

TheOREM 5. If two unitary spaces are conformal and the affinor $C_{\alpha \mu^{*} \nu}^{\cdots \beta}$ is a conformal invariant, then the spaces are $k$-spaces.

From (4.8), we find that the condition (4.13) is equivalent to the two conditions

$$
\begin{aligned}
& \partial_{\lambda^{*}} q_{\mu}=\partial_{\mu} q_{\lambda^{*}}, \text { conj., } \\
& \partial_{\mu} q_{\lambda^{*}}=\rho^{\prime} a_{\mu \lambda^{*}}, \text { conj. }
\end{aligned}
$$

From (3.8), we find

$$
\partial_{\mu} \partial_{\lambda^{*}} \log \sigma=2 \rho^{\prime} a_{\mu \lambda^{*}}, \text { conj., }
$$

where $\sigma$ is real. Hence our theorem is proved.

UNIVERSITY OF TEXAS,

Austin, Tex. 